\title{
Accelerated Methods for the SOCP-relaxed Component-based Distributed Optimal Power Flow
}

\author{
Sleiman Mhanna, MIEEE, Gregor Verbič, Senior MIEEE, and Archie C. Chapman, MIEEE
}

\begin{abstract}
In light of the increased focus on distributed methods, this paper proposes two accelerated subgradient methods and an adaptive penalty parameter scheme to speed-up the convergence of ADMM on the component-based dual decomposition of the second-order cone programming (SOCP) relaxation of the OPF. This work is the first to apply an adaptive penalty parameter method along with an accelerated subgradient method together in one scheme for distributed OPF. This accelerated scheme is demonstrated to reach substantial speed-ups, as high as $87 \%$, on real-world test systems with more than 9000 buses, as well as on other difficult test cases.
\end{abstract}

Index Terms-Accelerated methods, adaptive ADMM, component-based dual decomposition, distributed OPF.

\section{NOTATION}

\section{A. Input data and operators}

$\mathcal{B} \quad$ Set of buses in the power network.

$\mathcal{B}_{i} \quad$ Set of buses connected to bus $i$.

$b_{i}^{\text {sh }} \quad$ Shunt susceptance (p.u.) at bus $i$.

$g_{i}^{\text {sh }} \quad$ Shunt conductance (p.u.) at bus $i$.

$b_{i j}^{\text {ch }} \quad$ Charging susceptance (p.u.) in the $\pi$-model of line $i j$.

$c 0_{g i} \quad$ Constant coefficient $(\$)$ term of generator $g$ 's cost function.

$c 1_{g i} \quad$ Coefficient $(\$ / \mathrm{MW})$ of the linear term of generator $g$ 's cost function.

$c 2_{g i} \quad$ Coefficient $\left(\$ / \mathrm{MW}^{2}\right)$ of the quadratic term of generator $g$ 's cost function.

$\mathcal{G} \quad$ Set of all generators $(g, i)$ in the power network such that $g$ is the generator and $i$ is the bus connected to it.

$\mathcal{G}_{i} \quad$ Set of all generators connected to bus $i$.

$\mathrm{j} \quad$ Imaginary unit.

$\mathcal{L} \quad$ Set of all branches $i j$ where $i$ is the "from" bus.

$\mathcal{L}_{t} \quad$ Set of all branches $j i$ where $j$ is the "to" bus.

$p_{i}^{\mathrm{d}} / q_{i}^{\mathrm{d}} \quad$ Active/reactive power demand (MW/MVAr) at bus $i$.

$\bar{s}_{i j} \quad$ Apparent power rating (MVA) of line $i j$.

$\underline{\theta}_{i j}^{\Delta} \quad$ Lower limit of the difference of voltage angles of buses $i$ and $j$.

$\bar{\theta}_{i j}^{\Delta} \quad$ Upper limit of the difference of voltage angles of buses $i$ and $j$.

$\theta_{i}^{\text {shift }}$ Phase shift (Radians) of phase shifting transformer connected between buses $i$ and $j\left(\theta_{i}^{\text {shift }}=\right.$ 0 for a transmission line).

$\tau_{i j} \quad$ Tap ratio magnitude of phase shifting transformer connected between buses $i$ and $j\left(\tau_{i j}=1\right.$ for a transmission line).
$T_{i j} \quad$ Complex tap ratio of a phase shifting transformer $\left(T_{i j}=\tau_{i j} \mathrm{e}^{\mathrm{j} \theta_{i}^{\text {shitt }}}\right)$.

$Y_{i j} \quad$ Series admittance (p.u.) in the $\pi$-model of line $i j$.

$\Im\{\bullet\} \quad$ Imaginary value operator.

$\Re\{\bullet\} \quad$ Real value operator.

$\bullet / \bar{\bullet} \quad$ Minimum/maximum magnitude operator.

$\mid \bullet \quad$ Magnitude operator/Cardinality of a set.

-* Conjugate operator.

$k \quad$ Iteration number.

$\rho \quad$ ADMM penalty parameter.

B. Decision variables

$p_{g i} / q_{g i} \quad$ Active/reactive power (MW/MVAr) generation of generator $g$ at bus $i$.

$p_{g i_{(i)}} \quad$ Duplicate of $p_{g i}$ at bus $i$.

$q_{g i(i)} \quad$ Duplicate of $q_{g i}$ at bus $i$.

$p_{i j} / q_{i j} \quad$ Active/reactive power (MW/MVAr) flow along branch $i j$.

$p_{i j_{(i)}} \quad$ Duplicate of $p_{i j}$ at bus $i$.

$q_{i j_{(i)}} \quad$ Duplicate of $q_{i j}$ at bus $i$.

$V_{i} \quad$ Complex phasor voltage (p.u.) at bus $i\left(V_{i}=\right.$ $\left.\left|V_{i}\right| \angle \theta_{i}=v_{i} \angle \theta_{i}\right)$.

$\lambda \quad$ Vector of Lagrange multipliers.

\section{INTRODUCTION}

Up to this day, most optimization and control algorithms in power systems, such as the optimal power flow (OPF), are computed in a centralized fashion. With the increasing penetration of distributed energy resources however, the feasibility of the centralized computation paradigm is at stake for four main reasons. First, collecting all the required information from these DERs to centrally compile an OPF problem instance entails substantial communication overhead. Second, this information is private to the owners of these DERs and accessing it would raise privacy concerns. Third, most of these DERs require the use of mixed-integer variables to model them accurately. Their presence results in a mixed-integer nonlinear program (MINLP), which further increases the computational complexity of the AC OPF problem. Therefore, the resulting large-scale MINLP may be intractable if solved centrally to optimality. Fourth, centralized schemes are vulnerable to single point of failure or attack. To this end, an increased attention is given to distributed methods as they can be scalable, privacy preserving and robust to single point of failure.

There is a plethora of existing works on distributed OPF. These can be broadly classified into three categories, dual decomposition methods, optimality conditions decomposition 
(OCD) methods and sparse semidefinite programming (SDP) decomposition methods (see [1] and [2] for a review). The dual decomposition techniques underlying the dual-decompositionbased distributed OPF methods in the literature can in turn be classified into two categories: region-based decompositions and component-based decompositions. The focus of this study revolves around the latter decomposition techniques because they can distribute the computation across every component in the network (generators, transformers, loads, buses, transmission lines etc.) and are flexible enough to incorporate discrete decision variables to suit a wide variety of optimization applications in power systems and future grids. The result of the component-based dual decomposition is a consensus problem that can be solved in a distributed fashion using ADMM. ADMM was first introduced in [3] and its convergence was studied in [4]-[6]. ADMM is a highly desirable scheme to solve the distributed OPF problem because of its simplicity. However, its practical performance is poor when the problem is ill-conditioned or when high accuracy is required. The convergence of ADMM is also sensitive to the choice of penalty parameter.

Against this background, and motivated by the electricity industry's real-time decision-making applications, this paper proposes and compares three methods for accelerating the convergence of ADMM on the component-based dual decomposition of the second-order cone programming (SOCP) relaxation of the OPF. In more detail, the first method, called over-relaxed ADMM, is a popular variant of ADMM. This method is analyzed in [6] and [7], and is shown in [8] and [9] to improve the convergence of ADMM. Like the "vanilla" ADMM, this method is distributed, as each component communicates with its immediate neighbours, and there is no centralized controller. The second method is an adaptation of the predictor-corrector-type acceleration with restart scheme proposed in [10] (and later in [11]) for ADMM with weakly convex objectives, which is itself an adaptation of an optimal accelerated gradient descent method initially proposed by Nesterov [12]. However, in contrast to the over-relaxed ADMM method, this scheme is no longer fully distributed, as the restart rule relies on a combined residual which requires a central controller to compute the global primal and dual residuals. Moreover, since the convergence of ADMM is highly sensitive to the choice of penalty parameters, the third method is an adaptive consensus ADMM in which the penalty parameters are automatically tuned without a central oversight, which is suitable for dynamic network topologies underlying distributed consensus problems. Specifically, adaptive consensus ADMM sets the penalty parameters for each consensus constraint based on the relative magnitudes of the local primal and dual residuals. This method is inspired by the residual balancing scheme, and is demonstrated to achieve substantial speed-ups and relative insensitivity to the initial penalty parameter values and ill-conditioning. More interestingly, the convergence of the first two algorithms is further improved by overlaying them with the adaptive consensus ADMM scheme.

In light of recent promises of recovering feasible solutions from the SOCP relaxation of the OPF [13], the accelerated methods developed in this work are demonstrated on the SOCP-relaxed OPF for various good reasons. First, the SOCPrelaxed OPF is convex, which entails that applying ADMM to solve it comes with convergence guarantees. Another reason for working on the SOCP-relaxed OPF is that this relaxation is shown to be exact in radial networks under some mild conditions [14], [15]. Moreover, in mesh networks, on top of achieving small optimality gaps on many real-world test systems [16], the SCOP-relaxed OPF can be strengthened even further by bound tightening techniques and tight convex hulls [17]-[19]. A tightened SOCP-relaxed OPF in mesh networks is also used in [13] and its solution is used as an initial point for a proposed alternative convex optimization (ACP) OPF algorithm to recover a feasible solution. This ACP algorithm first formulates the OPF problem as a difference-of-convex programming (DCP) problem, then solves the DCP problem by penalty convex-concave procedure (CCP) iteratively. Finally, the component-based dual decomposition applied to the SOCP-relaxed OPF relishes closed-form solutions for the bus and generator subproblems. Problems with closed-form solutions are faster to compute compared to when they are solved using a numerical solver.

\section{A. Contributions of this work}

In summary, this paper advances the state of the art in the following ways:

- This paper is the first to apply an adaptive penalty parameter method along with an accelerated subgradient method together in one scheme for distributed OPF.

- The methods are implemented on real-world test systems [20] and other difficult test cases from NESTA v6 [16], and are demonstrated to reach substantial speed-ups, as high as $88 \%$.

The methods developed is this paper are readily transferable to other applications in power systems that are based on OPF, such as security constrained unit commitment (SCUC) with contingency constraints and multiple transmission system operators (TSOs), stochastic OPF, probabilistic OPF, and multiperiod OPF with demand response (DR), to name a few.

\section{THE OPF PROBLEM}

In a power network, the OPF problem consists of finding the least-cost dispatch of power from generators to satisfy the load at all buses in a way that is governed by physical laws, such as Ohm's Law and Kirchhoff's Law, and other technical restrictions, such as transmission line thermal limit constraints. By letting

$$
w_{i}=\left|V_{i}\right|^{2}, w_{i j}^{\mathrm{r}}=\Re\left\{V_{i} V_{j}^{*}\right\}, w_{i j}^{\mathrm{i}}=\Im\left\{V_{i} V_{j}^{*}\right\},
$$

the alternative formulation of the OPF problem can be written as

$$
\underset{\substack{p_{g i}, q_{g i}, w_{i}, w_{i j}^{\mathrm{r}}, w_{i j}^{\mathrm{i}}, \theta_{i}, p_{i j}, q_{i j}, p_{j i}, q_{j i}}}{\operatorname{minimi}} \sum_{(g, i) \in \mathcal{G}} f_{g i}\left(p_{g i}\right)
$$

subject to 


$$
\begin{array}{crr}
\underline{p}_{g i} \leq p_{g i} \leq \bar{p}_{g i}, & (g, i) \in \mathcal{G} \\
\underline{q}_{g i} \leq q_{g i} \leq \bar{q}_{g i}, & (g, i) \in \mathcal{G} \\
\left|\underline{V}_{i}\right|^{2} \leq w_{i} \leq\left|\bar{V}_{i}\right|^{2}, & i \in \mathcal{B} \\
\underline{\theta}_{i j}^{\Delta} \leq \theta_{i}-\theta_{j} \leq \bar{\theta}_{i j}^{\Delta}, & (i, j) \in \mathcal{L} \\
\sum_{(g, i) \in \mathcal{G}} p_{g i}-p_{i}^{\mathrm{d}}=\sum_{j \in \mathcal{B}_{i}} p_{i j}+g_{i}^{\mathrm{sh}} w_{i}, & i \in \mathcal{B} \\
\sum_{(g, i) \in \mathcal{G}} q_{g i}-q_{i}^{\mathrm{d}}=\sum_{j \in \mathcal{B}_{i}} q_{i j}-b_{i}^{\mathrm{sh}} w_{i}, & & \\
p_{i j}=g_{i j}^{\mathrm{c}} w_{i}-g_{i j} w_{i j}^{\mathrm{r}}+b_{i j} w_{i j}^{\mathrm{i}}, & (i, j) \in \mathcal{B} \\
q_{i j}=b_{i j}^{\mathrm{c}} w_{i}-b_{i j} w_{i j}^{\mathrm{r}}-g_{i j} w_{i j}^{\mathrm{i}}, & (i, j) \in \mathcal{L} \\
p_{j i}=g_{j i}^{\mathrm{c}} w_{j}-g_{j i} w_{i j}^{\mathrm{r}}-b_{j i} w_{i j}^{\mathrm{i}}, & (i, j) \in \mathcal{L} \\
q_{j i}=b_{j i}^{\mathrm{c}} w_{j}-b_{j i} w_{i j}^{\mathrm{r}}+g_{j i} w_{i j}^{\mathrm{i}}, & (i, j) \in \mathcal{L} \\
\left(w_{i j}^{\mathrm{r}}\right)^{2}+\left(w_{i j}^{\mathrm{i}}\right)^{2}=w_{i} w_{j}, & (i, j) \in \mathcal{L} \\
\theta_{j}-\theta_{i}=\operatorname{atan}_{2} 2\left(w_{i j}^{\mathrm{i}}, w_{i j}^{\mathrm{r}}\right), & (i, j) \in \mathcal{L} \\
\sqrt{p_{i j}^{2}+q_{i j}^{2} \leq \bar{s}_{i j},} \quad(i, j) \in \mathcal{L} \cup \mathcal{L}{ }_{t}
\end{array}
$$

where, $g_{i j}^{\mathrm{c}}:=\Re\left\{\frac{Y_{i j}^{*}-\mathrm{j} \frac{b_{i j}^{\mathrm{ch}}}{2}}{\left|T_{i j}\right|^{2}}\right\}, b_{i j}^{\mathrm{c}}:=\Im\left\{\frac{Y_{i j}^{*}-\mathrm{j} \frac{b_{i j}^{\mathrm{ch}}}{2}}{\left|T_{i j}\right|^{2}}\right\}, g_{i j}:=$ $\Re\left\{\frac{Y_{i j}^{*}}{T_{i j}}\right\}, b_{i j}:=\Im\left\{\frac{Y_{i j}^{*}}{T_{i j}}\right\}, g_{j i}^{\mathrm{c}}:=\Re\left\{Y_{j i}^{*}-\mathrm{j} \frac{b_{j i}^{\mathrm{ch}}}{2}\right\}, b_{j i}^{\mathrm{c}}:=$ $\Im\left\{Y_{j i}^{*}-\mathrm{j} \frac{b_{j i}^{\mathrm{ch}}}{2}\right\}, g_{j i}:=\Re\left\{\frac{Y_{j i}^{*}}{T_{j i}^{*}}\right\}$ and $b_{j i}:=\Im\left\{\frac{Y_{j i}^{*}}{T_{j i}^{*}}\right\}$, and $f_{g i}\left(p_{g i}\right):=c 2_{g i}\left(p_{g i}\right)^{2}+c 1_{g i}\left(p_{g i}\right)+c 0_{g i}$. The OPF in (2) is a nonconvex nonlinear optimization problem that is proven to be NP-hard [21], [22]. The nonconvexities stem from equality constraint 21], which describes the boundary of a rotated second-order cone, and $(2 \mathrm{~m})$ which contains the nonconvex atan2 function. The SOCP relaxation of the OPF in (2) is obtained by ignoring (2m) and relaxing (21) to

$$
\left(w_{i j}^{\mathrm{r}}\right)^{2}+\left(w_{i j}^{\mathrm{i}}\right)^{2} \leq w_{i} w_{j},(i, j) \in \mathcal{L},
$$

which is the convex hull of (21).

\section{COMPONENT-BASED DUAL DECOMPOSITION AND ADMM}

A component-based separability can be bestowed on the SOCP relaxation of (2) by creating copies of the following variables

$$
\begin{aligned}
p_{g i} & =p_{g i_{(i)}}, & & (g, i) \in \mathcal{G}, \\
q_{g i} & =q_{g i_{(i)}}, & & (g, i) \in \mathcal{G}, \\
p_{i j} & =p_{i j_{(i)}}, & & (i, j) \in \mathcal{L} \cup \mathcal{L}_{t}, \\
q_{i j} & =q_{i j_{(i)}}, & & (i, j) \in \mathcal{L} \cup \mathcal{L}_{t}, \\
w_{i_{(i j)}} & =w_{i}, & & (i, j) \in \mathcal{L} \cup \mathcal{L}_{t},
\end{aligned}
$$

and the SOCP-relaxed OPF problem now becomes

$$
\underset{\boldsymbol{x}, \boldsymbol{z}, w_{i j}^{\mathrm{r}}, w_{i j}^{\mathrm{i}}}{\operatorname{minimize}} \sum_{(g, i) \in \mathcal{G}} f_{g i}\left(p_{g i}\right)
$$

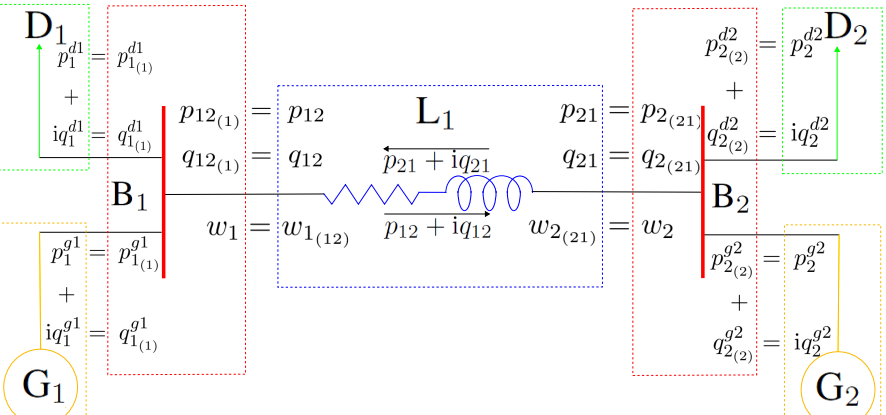

Figure 1. A 2-bus system showing the duplication of the coupling variables and the resulting component-based decomposition.

subject to (2b), (2c), (2n), (4)-(8)

$$
\begin{array}{r}
\left|\underline{V}_{i}\right|^{2} \leq w_{i_{(i j)}} \leq\left|\bar{V}_{i}\right|^{2}, \quad(i, j) \in \mathcal{L} \cup \mathcal{L}_{t} \\
\sum_{(g, i) \in \mathcal{G}} p_{g i_{(i)}}-p_{i}^{\mathrm{d}}=\sum_{j \in \mathcal{B}_{i}} p_{i j_{(i)}}+g_{i}^{\mathrm{sh}} w_{i}, \quad i \in \mathcal{B} \\
\sum_{(g, i) \in \mathcal{G}} q_{g i_{(i)}}-q_{i}^{\mathrm{d}}=\sum_{j \in \mathcal{B}_{i}} q_{i j_{(i)}}-b_{i}^{\mathrm{sh}} w_{i}, \quad i \in \mathcal{B} \\
p_{i j}=g_{i j}^{\mathrm{c}} w_{i_{(i j)}}-g_{i j} w_{i j}^{\mathrm{r}}+b_{i j} w_{i j}^{\mathrm{i}}, \quad(i, j) \in \mathcal{L} \\
q_{i j}=b_{i j}^{\mathrm{c}} w_{i_{(i j)}}-b_{i j} w_{i j}^{\mathrm{r}}-g_{i j} w_{i j}^{\mathrm{i}}, \quad(i, j) \in \mathcal{L} \\
p_{j i}=g_{j i}^{\mathrm{c}} w_{j_{(j i)}}-g_{j i} w_{i j}^{\mathrm{r}}-b_{j i} w_{i j}^{\mathrm{i}}, \quad(i, j) \in \mathcal{L} \\
q_{j i}=b_{j i}^{\mathrm{c}} w_{j_{(j i)}}-b_{j i} w_{i j}^{\mathrm{r}}+g_{j i} w_{i j}^{\mathrm{i}}, \quad(i, j) \in \mathcal{L} \\
\left(w_{i j}^{\mathrm{r}}\right)^{2}+\left(w_{i j}^{\mathrm{i}}\right)^{2} \leq w_{i_{(i j)}} w_{j(j i)}, \quad(i, j) \in \mathcal{L} \\
\tan \left(\underline{\theta}_{i j}^{\Delta} w_{i j}^{\mathrm{r}} \leq w_{i j}^{\mathrm{i}} \leq \tan \left(\bar{\theta}_{i j}^{\Delta}\right) w_{i j}^{\mathrm{r}}, \quad(i, j) \in \mathcal{L}\right.
\end{array}
$$

where

$$
\boldsymbol{x}:=\left[\left(p_{g i}, q_{g i}\right)_{(g, i) \in \mathcal{G}},\left(p_{i j}, q_{i j}, w_{i_{(i j)}}\right)_{(i, j) \in \mathcal{L} \cup \mathcal{L}_{t}}\right],
$$

and

$\boldsymbol{z}:=\left[\left(p_{i_{(i)}}^{g}, q_{i_{(i)}}^{g}\right)_{(g, i) \in \mathcal{G}},\left(p_{i j_{(i)}}, q_{i j_{(i)}}\right)_{(i, j) \in \mathcal{L} \cup \mathcal{L}_{t}},\left(w_{i}\right)_{i \in \mathcal{B}}\right]$.

This duplication of the coupling variables along with the resulting component-based decomposition are depicted in Figure 1 for a 2-bus system. Let $N_{\boldsymbol{x}}=N_{\boldsymbol{\lambda}}=2|\mathcal{G}|+3\left|\mathcal{L} \cup \mathcal{L}_{t}\right|$ and $N_{\boldsymbol{z}}=2|\mathcal{G}|+2\left|\mathcal{L} \cup \mathcal{L}_{t}\right|+|\mathcal{B}|$. Problem (9) is now of the general form

$$
\begin{aligned}
& \underset{\boldsymbol{x} \in \mathcal{X}, \boldsymbol{z} \in \mathcal{Z}}{\operatorname{minimize}} f(\boldsymbol{x})+g(\boldsymbol{z}) \\
& \text { subject to }
\end{aligned}
$$

where $f: \mathbf{R}^{N_{x}} \rightarrow \mathbf{R}$ and $g: \mathbf{R}^{N_{z}} \rightarrow \mathbf{R}$ are closed convex functions, $A$ is a $N_{\boldsymbol{\lambda}} \times N_{\boldsymbol{x}}$ identity matrix, $B \in \mathbf{R}^{N_{\boldsymbol{\lambda}} \times N_{\boldsymbol{z}}}$, $\left.c \in \mathbf{R}^{N_{\lambda}} 1\right] \mathcal{X}$ is the feasible set defined by constraints (9b), (9c), (9f)- $-9 \mathrm{jj}$ and $\mathcal{Z}$ is the feasible set defined by constraints (9d) and (9e). The augmented (partial) Lagrange function of (10) is written as

$$
L_{\rho}(\boldsymbol{x}, \boldsymbol{z}, \boldsymbol{\lambda}):=f(\boldsymbol{x})+g(\boldsymbol{z})+\boldsymbol{\lambda}^{T}(A \boldsymbol{x}+B \boldsymbol{z}-\boldsymbol{c})
$$

\footnotetext{
${ }^{1}$ Note that $\boldsymbol{c}=\mathbf{0}$ in this OPF case.
} 


$$
+\frac{\rho}{2}\|A \boldsymbol{x}+B \boldsymbol{z}-\boldsymbol{c}\|^{2},
$$

where $\rho>0$ is a penalty parameter and

$\boldsymbol{\lambda}:=\left[\left(\lambda_{p, i}^{g}, \lambda_{q, i}^{g}\right)_{(g, i) \in \mathcal{G}},\left(\lambda_{p_{i j}}, \lambda_{q_{i j}}, \lambda_{w_{i j}}\right)_{(i, j) \in \mathcal{L} \cup \mathcal{L}_{t}}\right] \in \mathbf{R}^{N_{\boldsymbol{\lambda}}}$,

is the vector of dual variables associated with coupling constraints (10b). The augmented Lagrangian in 111 is not separable in terms of sets of variables $(\mathcal{X}$ and $\mathcal{Z})$. Nonetheless, ADMM can be used to decouple these sets of variables, by using alternate minimizations over these sets. In particular, given the current iterates $\left(\boldsymbol{x}^{k}, \boldsymbol{z}^{k}, \boldsymbol{\lambda}^{k}\right)$, ADMM generates a new iterate $\left(\boldsymbol{x}^{k+1}, \boldsymbol{z}^{k+1}, \boldsymbol{\lambda}^{k+1}\right)$ as follows

$$
\begin{aligned}
& \boldsymbol{x}^{k+1} \in \underset{\boldsymbol{x} \in \mathcal{X}}{\arg \min } L_{\rho}\left(\boldsymbol{x}, \boldsymbol{z}^{k}, \boldsymbol{\lambda}^{k}\right), \\
& \boldsymbol{z}^{k+1} \in \underset{\boldsymbol{z} \in \mathcal{Z}}{\arg \min } L_{\rho}\left(\boldsymbol{x}^{k+1}, \boldsymbol{z}, \boldsymbol{\lambda}^{k}\right), \\
& \boldsymbol{\lambda}^{k+1}=\boldsymbol{\lambda}^{k}+\rho\left(A \boldsymbol{x}^{k+1}+B \boldsymbol{z}^{k+1}-\boldsymbol{c}\right) .
\end{aligned}
$$

More specifically, generators now solve

$$
\begin{gathered}
D_{\rho, i}^{g}\left(\boldsymbol{\lambda}_{i}^{g, k}\right)=\underset{\boldsymbol{x}_{g i}}{\operatorname{minimize}} \sum_{g \in \mathcal{G}_{i}}\left(f_{g i}\left(p_{g i}\right)+\left\langle\boldsymbol{\lambda}_{i}^{g, k}, \boldsymbol{x}_{g i}\right\rangle\right. \\
\left.+\frac{\rho}{2}\left(\left(p_{g i}-p_{i_{(i)}}^{g, k}\right)^{2}+\left(q_{g i}-q_{i_{(i)}}^{g, k}\right)^{2}\right)\right) \\
\text { subject to (2b), (2c) }
\end{gathered}
$$

where $\boldsymbol{x}_{g i}:=\left[p_{g i}, q_{g i}\right]$ and $\boldsymbol{\lambda}_{g i}:=\left[\lambda_{p, g i}, \lambda_{q, g i}\right]$, and branches (transmission lines, transformers) solve

$$
\begin{gathered}
D_{\rho, i j}^{1}\left(\boldsymbol{\lambda}_{i j}^{k}, \boldsymbol{\lambda}_{j i}^{k}\right)=\underset{\boldsymbol{x}_{i j}^{1}}{\operatorname{minimize}}\left\{\left\langle\left[\boldsymbol{\lambda}_{i j}^{k}, \boldsymbol{\lambda}_{j i}^{k}\right], \boldsymbol{x}_{i j}^{1}\right\rangle+\right. \\
\sum_{(l, m) \in\{(i, j) \cup(j, i)\}} \frac{\rho}{2}\left(\left(w_{l}^{k}-w_{l(l m)}\right)^{2}+\right. \\
\left.\left.\left(p_{l m}-p_{l m_{(l)}}^{k}\right)^{2}+\left(q_{l m}-q_{l m_{(l)}}^{k}\right)^{2}\right)\right\}
\end{gathered}
$$

subject to $9 \mathrm{c}$, , $9 \mathrm{f}-9 \mathrm{k},(2 \mathrm{n}$,

where $\boldsymbol{x}_{i j}^{1}:=\left[p_{i j}, q_{i j}, w_{i_{(i j)}}, p_{j i}, q_{j i}, w_{j_{(j i)}}\right], \quad \boldsymbol{\lambda}_{i j}:=$ $\left[\lambda_{p_{i j}}, \lambda_{q_{i j}}, \lambda_{w_{i j}}\right]$ and $\lambda_{j i}:=\left[\lambda_{p_{j i}}, \lambda_{q_{j i}}, \lambda_{w_{j i}}\right]$. On the other hand, buses solve

$$
\begin{aligned}
& D_{\rho, i}^{\mathrm{b}}\left(\left(\boldsymbol{\lambda}_{i}^{g, k}\right)_{g \in \mathcal{G}_{i}},\left(\boldsymbol{\lambda}_{i j}^{k}\right)_{j \in \mathcal{B}_{i}}\right)= \\
& \operatorname{minimize}_{\boldsymbol{z}_{i}}\left\{\sum _ { g \in \mathcal { G } _ { i } } \left(-\left\langle\boldsymbol{\lambda}_{g i}^{k},\left[p_{g i_{(i)}}, q_{g i_{(i)}}\right]\right\rangle+\right.\right. \\
&\left.\frac{\rho}{2}\left(\left(p_{g i}^{k+1}-p_{g i_{(i)}}\right)^{2}+\left(q_{g i}^{k+1}-q_{g i_{(i)}}\right)^{2}\right)\right)+ \\
& \sum_{j \in \mathcal{B}_{i}}\left(-\left\langle\boldsymbol{\lambda}_{i j}^{k},\left[p_{i j_{(i)}}, q_{i j_{(i)}}, w_{i}\right]\right\rangle+\right. \\
& \frac{\rho}{2}\left(\left(p_{i j}^{k+1}-p_{i j_{(i)}}\right)^{2}+\left(q_{i j}^{k+1}-q_{i j_{(i)}}\right)^{2}\right)+ \\
&\left.\left.\left(\left(w_{i}-w_{i_{(i j)}}^{k+1}\right)^{2}\right)\right)\right\},
\end{aligned}
$$

subject to 9d, $9 \mathrm{e}$.

where

$$
\boldsymbol{z}_{i}:=\left[\left(p_{g i_{(i)}}, q_{g i_{(i)}}\right)_{(g, i) \in \mathcal{G}}, w_{i},\left(p_{i j_{(i)}}, q_{i j_{(i)}}\right)_{j \in \mathcal{B}_{i}}\right] .
$$

The advantages of applying the component-based decomposition to the alternative OPF formulation are that buses and generators admit closed-form solutions (see [23]). The primal residuals are defined as

$$
\boldsymbol{r}^{k+1}=\left[r_{1}^{k+1}, \ldots, r_{N_{\boldsymbol{\lambda}}}^{k+1}\right]=A \boldsymbol{x}^{k+1}+B \boldsymbol{z}^{k+1}-\boldsymbol{c},
$$

and the dual residuals as

$$
\boldsymbol{s}^{k+1}=\left[s_{1}^{k+1}, \ldots, s_{N_{\boldsymbol{\lambda}}}^{k+1}\right]=\rho A^{T} B\left(\boldsymbol{z}^{k+1}-\boldsymbol{z}^{k}\right) .
$$

The algorithm in (12) is terminated when

$$
\left\|\boldsymbol{r}^{k}\right\| \leq \epsilon^{\text {pri }} \text { and }\left\|s^{k}\right\| \leq \epsilon^{\text {dual }},
$$

where $\epsilon^{\text {pri }}$ and $\epsilon^{\text {dual }}$ are feasibility tolerances which are chosen using an absolute and relative criterion (see [24]) as follows

$$
\begin{aligned}
\epsilon^{\mathrm{pri}} & =\sqrt{N_{\boldsymbol{\lambda}}} \epsilon^{\mathrm{abs}}+\epsilon^{\mathrm{rel}} \max \left\{\left\|A \boldsymbol{x}^{k}\right\|,\left\|B \boldsymbol{z}^{k}\right\|,\|\boldsymbol{c}\|\right\}, \\
\epsilon^{\mathrm{dual}} & =\sqrt{N_{\boldsymbol{x}}} \epsilon^{\mathrm{abs}}+\epsilon^{\mathrm{rel}}\left\|A^{T} \boldsymbol{\lambda}^{k}\right\|,
\end{aligned}
$$

where $\epsilon^{\mathrm{abs}}>0$ and $\epsilon^{\mathrm{rel}}>0$ are absolute and relative tolerances respectively. The values of $\boldsymbol{r}^{k}$ and $\boldsymbol{s}^{k}$ indicate how distant the iterates are from a solution 2 If the sets $\mathcal{X}$ and $\mathcal{Z}$ are convex (which is the case for the SOCP-relaxed OPF) and problem (10) is feasible, ADMM is guaranteed to converge to an optimal point [24]. The main objective of this paper is to establish accelerated variants for the algorithm in (12) to ensure that these residuals decay quickly. To this end, this paper presents two accelerated methods in Section [IV and an adaptive penalty parameter method in Section $\mathrm{V}$.

\section{ACCELERATED METHODS}

Because of its simplicity, ADMM is a desirable way to solve (10). However, since the OPF problem in high voltage transmission systems is inherently poorly conditioned (high inductance-to-resistance ratio), ADMM exhibits a poor performance, especially when high precision is required. In fact, ADMM is shown to have a convergence rate of $\mathcal{O}\left(\frac{1}{k}\right)$ [26]. This section presents two accelerated variants of ADMM, adapted to the SOCP-relaxed OPF setting.

\section{A. Over-relaxed ADMM}

A popular variant of the algorithm in (12) is the over-relaxed ADMM, which introduces a relaxation parameter $\alpha \in(0,2)$ and replaces each instance of $A \boldsymbol{x}^{k+1}$ in the $\boldsymbol{z}$ and $\boldsymbol{\lambda}$ updates in (12) with

$$
\alpha A \boldsymbol{x}^{k+1}-(1-\alpha)\left(B \boldsymbol{z}^{k}-c\right)
$$

${ }^{2}$ Note that the vanilla ADMM requires a central controller to check for convergence. However, if a central controller is unavailable, ADMM can in practice be run continuously over a fixed period of time, with no stopping criterion (see [25]), which makes the scheme fully distributed. 

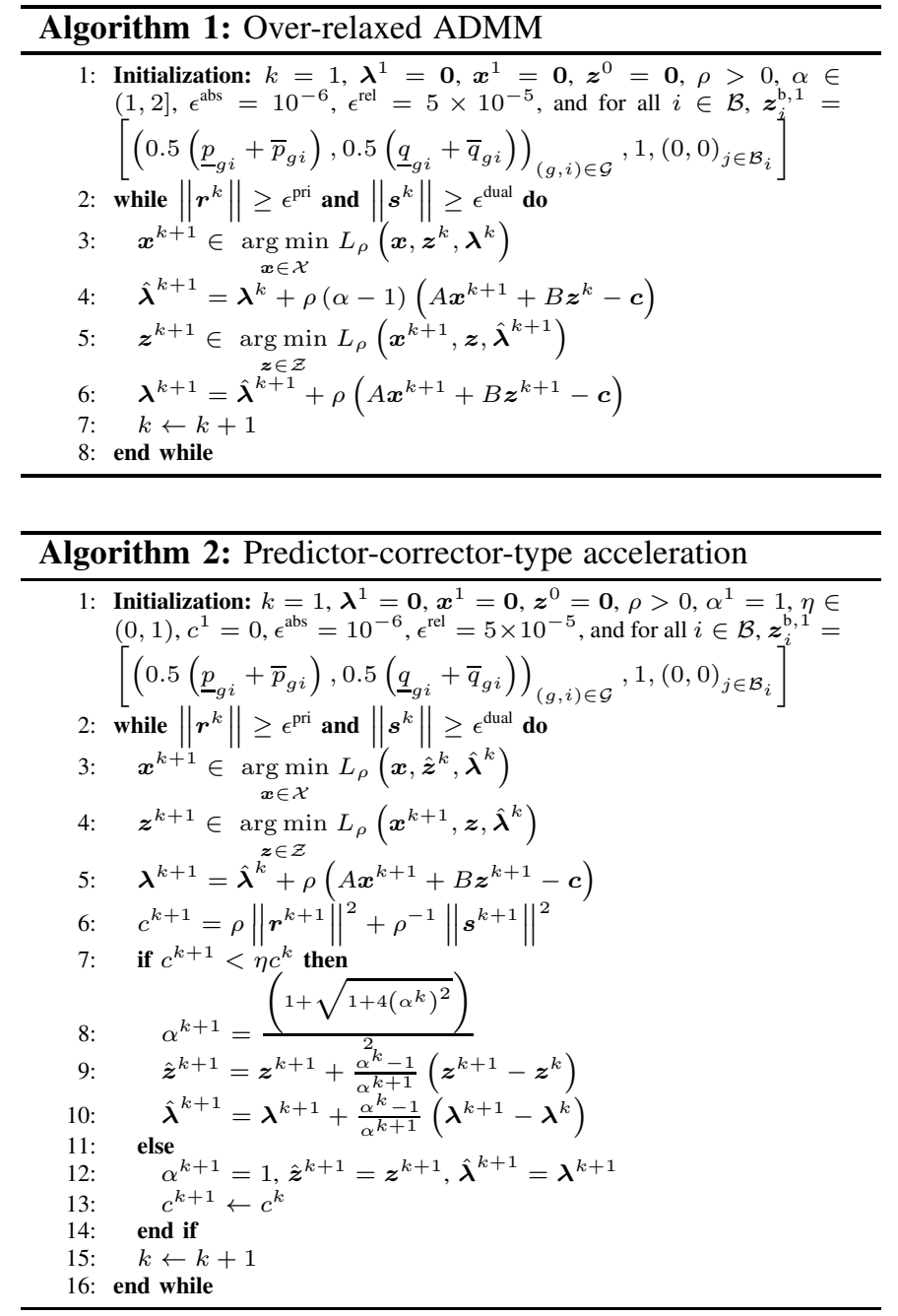

The over-relaxed ADMM is described in Algorithm 1 When $\alpha=1$, Algorithm 1 and the vanilla ADMM in (12) coincide. This method is analyzed in [6] and [7], and empirical studies in [8] show that over-relaxation with $\alpha \in[1.5,1.8]$ is more conducive to faster convergence. This method is also shown in [9] to improve convergence on quadratic problems.

\section{B. Nesterov-type acceleration}

In this section, the accelerated variant of ADMM is described in Algorithm 2 The linchpin of this accelerated method is the predictor-corrector-type acceleration step containing an over-relaxation step, initially proposed by Nesterov for gradient descent methods [12]. In this case the primal residual is unchanged but the dual residual is changed to

$$
\boldsymbol{s}^{k+1}=\rho A^{T} B\left(\boldsymbol{z}^{k+1}-\hat{\boldsymbol{z}}^{k}\right),
$$

as in [10]. This method was originally aimed at accelerating gradient descent-type (first-order) methods and was shown by Nesterov to achieve a complexity $\mathcal{O}\left(\frac{1}{k^{2}}\right)$, a rate which is proven to be optimal [12], [27]. This method was first adapted to ADMM in [10] and is further modified is this paper to suit the SOCP-relaxed OPF problem in which $f$ and $g$ are minimized over $x \in \mathcal{X}$ and $z \in \mathcal{Z}$ instead of over $x \in \mathbf{R}^{N_{x}}$ and $z \in \mathbf{R}^{N_{z}}$, respectively. More specifically, setting $\hat{\boldsymbol{z}}^{k+1}=\boldsymbol{z}^{k+1}$ and $\hat{\boldsymbol{\lambda}}^{k+1}=\boldsymbol{\lambda}^{k+1}$ in the restart step on lines 12-13 of Algorithm 2 results in a faster convergence compared to $\hat{\boldsymbol{z}}^{k+1}=\boldsymbol{z}^{k}$ and $\hat{\boldsymbol{\lambda}}^{k+1}=\boldsymbol{\lambda}^{k}$.

The convergence rate of $\mathcal{O}\left(\frac{1}{k^{2}}\right)$ can no longer be proven for Algorithm 2 because of the restart rule; nonetheless Algorithm 2 is still guaranteed to converge because problem (9) is convex $3^{3}$ The restart rule is needed in this OPF setting because the residuals do not decrease monotonically. Algorithm 2 s acceleration potential is demonstrated empirically in Section VI. In all the numerical evaluations in Section VI, $\eta=0.999$ is used, as in [10]. Note that in contrast to the over-relaxed ADMM method in the previous section, the predictor-corrector-type acceleration scheme is no longer fully distributed, as updating the combined residual $c^{k+1}$ on line 6 of Algorithm 2 requires the global computation of the primal and dual residuals.

\section{ADAPTIVE PENALTY PARAMETER}

The convergence of ADMM is in practice sensitive to the choice of $\rho$. A natural extension is to allow this parameter to vary at each iteration $k$. One such varying penalty parameter scheme, proposed in [28] and [29], updates $\rho$ based on the relative magnitudes of the primal and dual residuals as follows

$$
\rho^{k+1}:= \begin{cases}\rho^{k}\left(1+\tau^{\text {incr }}\right) & \text { if }\left\|\boldsymbol{r}^{k+1}\right\|>\mu^{\text {incr }}\left\|\boldsymbol{s}^{k+1}\right\|, \\ \rho^{k}\left(1+\tau^{\mathrm{dec}}\right)^{-1} & \text { if }\left\|\boldsymbol{s}^{k+1}\right\|>\mu^{\text {decr }}\left\|\boldsymbol{r}^{k+1}\right\|, \\ \rho^{k} & \text { otherwise, }\end{cases}
$$

where $\tau^{\text {incr }}>0, \tau^{\text {dec }}>0, \mu^{\text {incr }}>1$ and $\mu^{\text {decr }}>1$ are parameters. The founding premise of this scheme is to balance the primal and dual residual magnitudes to within a factor of $\mu$ of one another as they both converge to zero. This varying penalty scheme is shown to improve the convergence of ADMM, in addition to making it less dependent on the initial choice of this parameter. However, this scheme is not suitable for a distributed setting as it relies on a central controller to compute the global residuals. Nonetheless, by introducing $\boldsymbol{\rho}^{k+1}:=\left[\rho_{1}^{k+1}, \ldots, \rho_{N_{\boldsymbol{\lambda}}}^{k+1}\right]$, where $\rho_{p}^{k+1}$ is a penalty parameter associated with each consensus constraint, the residual balancing scheme in (23) can be extended to the distributed setting as follows

$$
\rho_{p}^{k+1}:= \begin{cases}\rho_{p}^{k}\left(1+\tau^{\mathrm{incr}}\right) & \text { if }\left|r_{p}^{k+1}\right|>\mu^{\mathrm{incr}}\left|s_{p}^{k+1}\right|, \\ \rho_{p}^{k+1}\left(1+\tau^{\mathrm{dec}}\right)^{-1} & \text { if }\left|s_{p}^{k+1}\right|>\mu^{\mathrm{decr}}\left|r_{p}^{k+1}\right|, \\ \rho_{p}^{k+1} & \text { otherwise, } \\ \quad p \in\left\{1, \ldots, N_{\boldsymbol{\lambda}}\right\},\end{cases}
$$

where $r_{p}^{k+1}$ and $s_{p}^{k+1}$ are the local primal and dual residuals 4 The generalization of (23) to distributed consensus problems

\footnotetext{
${ }^{3}$ See [10] for the proof.

${ }^{4}$ These local primal and dual residuals can be computed at the corresponding buses.
} 


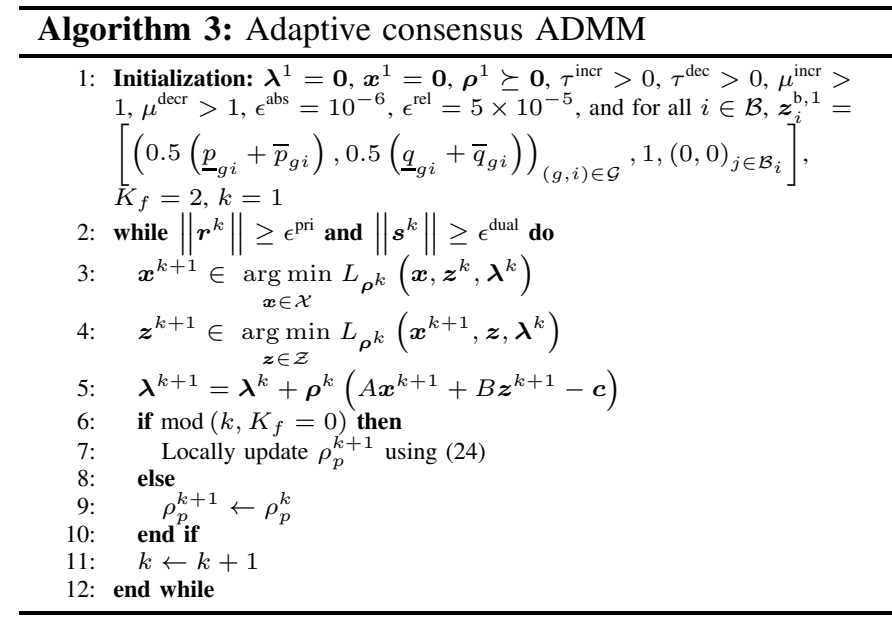

has been explored in [30]. However, in contrast to the method in [30], the update scheme (24) in this work is executed every $K_{f}=2$ iterations to avoid oscillations due to frequent changes in $\rho_{p}^{k+1}$. The end result, shown in Algorithm 3 is an adaptive scheme that automatically tunes penalty parameters without central oversight, which is suitable for dynamic network topologies underlying distributed consensus problems. In practice, a system operator can conduct off-line vanilla ADMM simulations to get a ballpark figure of the penalty parameter values that lead to a fast convergence and these are then used as an initialization for Algorithm 3. In all the simulations in Section VI below, the parameters of Algorithm 3 are set to $\tau^{\text {incr }}=1, \tau^{\text {decr }}=0.5, \mu^{\text {incr }}=10$ and $\mu^{\text {decr }}=100$.

\section{NUMERICAL EVALUATION}

The two accelerated methods along with the adaptive ADMM scheme are evaluated on MATPOWER's case 5 [31] and on PEGASE test systems with up to 9241 buses [20]. The versatility and robustness of the methods are also assessed by evaluating them on NESTA's Case_24_ieee_rts_sad (small angle difference conditions) [16], a test case which is particularly difficult to solve using the vanilla ADMM in (12), as shown in the column 2 of Table $\coprod^{5}$ The simulations are all conducted on a computing platform with 10 Intel Xeon E5-2687W v3 CPUs at 3.10GHz, 64-bit operating system, and 128GB RAM. In all simulations, OPTI [32] is used as a frontend modelling language along with IPOPT v3.12.5 [33] as a backend solver for the convex branch subproblems in (14). Generator and bus subproblems are convex and admit closedform solutions (see [23]). In Algorithms 1 and 2, $\rho=10$ is used for the active and reactive power consensus constraints (4)-(7) and $\rho=100$ is used for the voltage consensus constraints (8), i.e. $\boldsymbol{\rho}:=\left[(10)_{2|\mathcal{G}|+2\left|\mathcal{L} \cup \mathcal{L}_{t}\right|},(100)_{\left|\mathcal{L} \cup \mathcal{L}_{t}\right|}\right]$. This specific parameter tuning, which sets a higher value for the voltage consensus constraints, is shown in [2] to significantly improve the convergence of vanilla ADMM and can also

\footnotetext{
${ }^{5}$ The NESTA test cases are designed specifically to incorporate key network parameters such as line thermal limits and small angle differences, which are critical in optimization applications.
}

mean the difference between convergence and divergence in some cases. The performance of Algorithm 1 for the three different values of $\alpha$ is listed in columns 2, 3 and 4 of Table [] whereas the performance of Algorithm [2] is listed in column 8. The adaptive consensus ADMM method (Algorithm 3) is overlaid with the over-relaxed ADMM (Algorithm 11 and the resulting scheme's performance is shown in columns 5, 6 and 7 of Table I for three different values of $\alpha$ and for $\boldsymbol{\rho}^{1}:=\left[(10)_{2|\mathcal{G}|+2\left|\mathcal{L} \cup \mathcal{L}_{t}\right|},(100)_{\left|\mathcal{L} \cup \mathcal{L}_{t}\right|}\right]$. The adaptive consensus ADMM method (Algorithm 3) is also overlaid with the predictor-corrector-type acceleration method (Algorithm 2) and the resulting scheme's performance is shown in columns 9 of Table I, which is also initialized with $\boldsymbol{\rho}^{1}:=\left[(10)_{2|\mathcal{G}|+2\left|\mathcal{L} \cup \mathcal{L}_{t}\right|},(100)_{\left|\mathcal{L} \cup \mathcal{L}_{t}\right|}\right]$. The second to last column in Table I shows the percentage speed-up of the fastest method relative to the vanilla ADMM (Algorithm 1 with $\alpha=1$ ) and the last column shows the maximum violation in the consensus constraints (4)-(8) at the termination of the fastest method.

The main observations from Table \ can be summarized as follows. The over-relaxed ADMM accelerates the convergence in all the cases for both $\alpha=1.5$ and $\alpha=1.8$, but without a clear-cut conclusion over which value of $\alpha$ is better in general. The predictor-corrector-type acceleration scheme also improves the convergence in most test cases. However, only when these accelerated subgradient schemes are overlaid with the adaptive ADMM method that substantial speed-ups are achieved. More specifically, the Fast \& Adaptive ADMM scheme generally exhibits the best performance but, in fact, the speed-up in the cases where the Fast \& Adaptive scheme is faster than the Over-relaxed \& Adaptive ADMM scheme is only marginal. The most notable acceleration is witnessed on NESTA's Case_24_ieee_rts_sad case on which the vanilla ADMM requires 26496 iterations to converge. The adaptive penalty parameter scheme reduces this iteration count to 3380 , which is almost 8 times faster. This observation, along with the fact that the Over-relaxed \& Adaptive ADMM scheme is fully distributed, makes it the most attractive of the four algorithms even for the plain case $(\alpha=1)$. This underscores the substantial contribution of the adaptive consensus ADMM to the overall speed-up.

\section{CONCLUSION}

This paper proposes and assesses two accelerated subgradient methods and an adaptive penalty parameter scheme for improving the convergence of ADMM on the component-based dual decomposition of the SOCP-relaxed OPF. Both accelerated subgradient methods are shown to improve convergence in most test cases but only when these accelerated subgradient schemes are overlaid with the adaptive ADMM that substantial speed-ups are achieved. This makes the adaptive ADMM scheme is a key contributor in substantially accelerating the convergence of ADMM. The end result, is a fast adaptive scheme that automatically tunes penalty parameters without central oversight, which is suitable for dynamic network topologies underlying distributed consensus problems. 
TABLE I

PERFORMANCE OF THE PROPOSED METHODS MEASURED IN THE NUMBER ITERATIONS TO CONVERGENCE (COLUMNS 2 TO 9). COLUMN 10 SHOWS THE PERCENTAGE SPEED-UP OF THE FASTEST METHOD AND THE LAST COLUMN SHOWS MAX $(\boldsymbol{r})$ AT THE TERMINATION OF THE FASTEST METHOD.

\begin{tabular}{|c|c|c|c|c|c|c|c|c|c|c|}
\hline & \multirow{2}{*}{\multicolumn{3}{|c|}{$\begin{array}{c}\text { Over-relaxed } \\
\text { (Alg. 1 }\end{array}$}} & \multirow{2}{*}{\multicolumn{3}{|c|}{$\begin{array}{l}\text { Over-relaxed \& Adaptive } \\
\text { (Alg. 1 with 3 }\end{array}$}} & \multirow{3}{*}{$\begin{array}{c}\text { Fast } \\
\text { (Alg. 2] } \\
\eta=0.999\end{array}$} & \multirow{3}{*}{$\begin{array}{c}\text { Fast \& Adaptive } \\
\text { (Alg. 2 with 3 } \\
\eta=0.999\end{array}$} & \multirow{3}{*}{$\begin{array}{c}\text { Speed-up } \\
(\%)\end{array}$} & \multirow{3}{*}{$\max (\boldsymbol{r})$} \\
\hline & & & & & & & & & & \\
\hline Test case & $\alpha=1$ & $\alpha=1.5$ & $\alpha=1.8$ & $\alpha=1$ & $\alpha=1.5$ & $\alpha=1.8$ & & & & \\
\hline Case_5 & 1,681 & 1,120 & 947 & 372 & 362 & 471 & 1,629 & 355 & 78.88 & $2.48 \mathrm{E}-04$ \\
\hline Case_89_PEGASE & 2,677 & 1,857 & 1,600 & 1,220 & 900 & 877 & 1,911 & 915 & 67.24 & $1.59 \mathrm{E}-03$ \\
\hline Case_1354_PEGASE & 723 & 645 & 696 & 494 & 586 & 972 & 818 & 467 & 35.41 & $9.70 \mathrm{E}-04$ \\
\hline Case_2869_PEGASE & 906 & 679 & 857 & 567 & 679 & 961 & 854 & 560 & 38.19 & $2.28 \mathrm{E}-03$ \\
\hline Case_9241_PEGASE & 2,737 & 3,700 & 10,000 & 820 & 10,000 & 10,000 & 2,868 & 737 & 73.07 & $7.78 \mathrm{E}-03$ \\
\hline Case_24_ieee_rts_sad & 26,496 & 17,665 & 14,721 & 3,380 & 4,024 & 5,369 & 24,563 & 3,404 & 87.24 & 4.89E-04 \\
\hline Case_29_ieee_rts_sad & 1,569 & 1,259 & 1,134 & 314 & 730 & 718 & 1,233 & 329 & 79.99 & $2.00 \mathrm{E}-03$ \\
\hline
\end{tabular}

\section{REFERENCES}

[1] D. K. Molzahn, F. Dorfler, H. Sandberg, S. H. Low, S. Chakrabarti, R. Baldick, and J. Lavaei, "A Survey of Distributed Optimization and Control Algorithms for Electric Power Systems," IEEE Transactions on Smart Grid, vol. 3053, no. c, pp. 1-1, 2017.

[2] S. Mhanna, A. C. Chapman, and G. Verbič, "Component-based dual decomposition methods for the OPF problem," Sustainable Energy, Grids and Networks, vol. 16, pp. 91-109, dec 2018.

[3] R. Glowinski and A. Marroco, "Sur l'Approximation, par Elements d'Ordre un, et la Resolution, par Penalisation-Dualité, d'une Classe de Problemes de Dirichlet non Lineares," Revue Française d'Automatique, Informatique, et Recherche Operationelle, vol. 9(R-2), no. 2, pp. 41-76, 1975.

[4] D. Gabay and B. Mercier, "A dual algorithm for the solution of nonlinear variational problems via finite element approximation," Computers \& Mathematics with Applications, vol. 2, no. 1, pp. 17-40, 1976.

[5] R. Glowinski and P. Le Tallec, Augmented Lagrangian and OperatorSplitting Methods in Nonlinear Mechanics. Society for Industrial and Applied Mathematics, jan 1989.

[6] J. Eckstein and D. P. Bertsekas, "On the Douglas-Rachford splitting method and the proximal point algorithm for maximal monotone operators," Mathematical Programming, vol. 55, pp. 293-318, 1992.

[7] R. Nishihara, L. Lessard, B. Recht, A. Packard, and M. Jordan, "A general analysis of the convergence of ADMM," in Proceedings of the 32nd International Conference on Machine Learning (ICML-15), D. Blei and F. Bach, Eds. JMLR Workshop and Conference Proceedings, 2015, pp. 343-352.

[8] J. Eckstein, "Parallel alternating direction multiplier decomposition of convex programs," Journal of Optimization Theory and Applications, vol. 80, no. 1, pp. 39-62, jan 1994.

[9] E. Ghadimi and A. Teixeira, "Optimal parameter selection for the alternating direction method of multipliers (ADMM): quadratic problems," IEEE Transactions on Automatic Control, vol. 60, no. 3, pp. 644-658, mar 2015

[10] T. Goldstein, B. O'Donoghue, S. Setzer, and R. Baraniuk, "Fast alternating direction optimization methods," SIAM Journal of Imaging Sciences, vol. 7, no. 3, pp. 1588-1623, 2014.

[11] M. Kadkhodaie, K. Christakopoulou, M. Sanjabi, and A. Banerjee, "Accelerated Alternating Direction Method of Multipliers," in Proceedings of the 21th ACM SIGKDD International Conference on Knowledge Discovery and Data Mining - KDD '15. New York, New York, USA: ACM Press, 2015, pp. 497-506.

[12] Y. Nesterov, Introductory Lectures on Convex Optimization, 2004, vol. 87.

[13] Z. Tian and W. Wu, "Recover Feasible Solutions for SOCP Relaxation of Optimal Power Flow Problems in Mesh Networks," aug 2017.

[14] M. Farivar and S. H. Low, "Branch flow model: Relaxations and convexification-part i," IEEE Transactions on Power Systems, vol. 28, no. 3, pp. 2554-2564, aug 2013.

[15] L. Gan, N. Li, U. Topcu, and S. H. Low, "Exact Convex Relaxation of Optimal Power Flow in Radial Networks," IEEE Transactions on Automatic Control, vol. 60, no. 1, pp. 72-87, jan 2015.

[16] C. Coffrin, D. Gordon, and P. Scott, "NESTA, the NICTA energy system test case archive," CoRR, vol. abs/1411.0359, 2014. [Online]. Available: http://arxiv.org/abs/1411.0359.
[17] C. Chen, A. Atamtürk, and S. S. Oren, "Bound Tightening for the Alternating Current Optimal Power Flow Problem," IEEE Transaction on Power Systems, vol. 31, no. 5, pp. 1-8, 2015.

[18] C. Coffrin, H. Hijazi, and P. Van Hentenryck, "Strengthening convex relaxations with bound tightening for power network optimization," in Principles and Practice of Constraint Programming, ser. Lecture Notes in Computer Science, G. Pesant, Ed. Springer International Publishing, 2015, vol. 9255, pp. 39-57.

[19] B. Kocuk, S. S. Dey, and X. A. Sun, "Strong SOCP relaxations for the optimal power flow problem," Operations Research, 2016.

[20] C. Josz, S. Fliscounakis, J. Maeght, and P. Panciatici, "AC power flow data in MATPOWER and QCQP format: iTesla, RTE snapshots, and PEGASE," arXiv preprint arXiv:1603.01533, 2016.

[21] D. Bienstock and A. Verma, "Strong NP-hardness of AC power flows feasibility," dec 2015.

[22] K. Lehmann, A. Grastien, and P. Van Hentenryck, "AC-Feasibility on Tree Networks is NP-Hard," IEEE Transactions on Power Systems, vol. 31, no. 1, pp. 798-801, jan 2016.

[23] Q. Peng and S. H. Low, "Distributed optimal power flow algorithm for radial networks, i: Balanced single phase case," IEEE Transactions on Smart Grid, pp. 1-11, to be published.

[24] S. Boyd, N. Parikh, E. Chu, B. Peleato, and J. Eckstein, "Distributed optimization and statistical learning via the alternating direction method of multipliers," Foundations and Trends $\AA$ in Machine Learning, vol. 3, no. 1, pp. 1-122, 2011.

[25] M. Kraning, E. Chu, J. Lavaei, and S. Boyd, "Dynamic network energy management via proximal message passing," Found. Trends Optim. vol. 1, no. 2, pp. 73-126, Jan. 2014.

[26] B. He and X. Yuan, "On the $\mathrm{O}(1 / \mathrm{t})$ Convergence Rate of Alternating Direction Method," SIAM Journal on Numerical Analysis, vol. 50, no. 2, pp. 700-709, 2012.

[27] Y. Nesterov, "Smooth minimization of non-smooth functions," Mathematical Programming, vol. 103, no. 1, pp. 127-152, may 2005.

[28] B. S. He, H. Yang, and S. L. Wang, "Alternating Direction Method with Self-Adaptive Penalty Parameters for Monotone Variational Inequalities," Journal of Optimization Theory and Applications, vol. 106, no. 2, pp. 337-356, aug 2000.

[29] S. L. WANG and L. Z. LIAO, "Decomposition Method with a Variable Parameter for a Class of Monotone Variational Inequality Problems," Journal of Optimization Theory and Applications, vol. 109, no. 2, pp. 415-429, may 2001.

[30] C. Song and V. Pavlovic, "Fast ADMM Algorithm for Distributed Optimization with Adaptive Penalty," Proceedings of the 30th Conference on Artificial Intelligence (AAAI 2016), pp. 1-11, jun 2016.

[31] R. Zimmerman, C. Murillo-Sánchez, and R. Thomas, "MATPOWER: Steady-state operations, planning, and analysis tools for power systems research and education," IEEE Transactions on Power Systems, vol. 26, no. 1, pp. 12-19, Feb 2011.

[32] J. Currie and D. I. Wilson, "OPTI: Lowering the Barrier Between Open Source Optimizers and the Industrial MATLAB User," in Foundations of Computer-Aided Process Operations, N. Sahinidis and J. Pinto, Eds., Savannah, Georgia, USA, 8-11 January 2012.

[33] A. Wächter and L. T. Biegler, "On the implementation of an interiorpoint filter line-search algorithm for large-scale nonlinear programming," Mathematical Programming, vol. 106, no. 1, pp. 25-57, 2006. 